\title{
Adolescência:
}

\section{ações e percepção dos médicos e enfermeiros do Programa Saúde da Família}

Rosângela Aparecida Pimenta Ferrari ${ }^{1}$

Zuleika Thomson ${ }^{2}$

Regina Melchior ${ }^{3}$

FERRARI, R.A.P.; THOMSON, Z.; MELCHIOR, R. Adolescence: actions and perceptions of doctors and nurses within the Family Healthcare Program. Interface - Comunic.,

Saúde, Educ., v.12, n.25, p.387-400, abr./jun. 2008.

Among the many activities that family healthcare doctors and nurses carry out, the present study had the aims of characterizing the programmed, preventive and interventional actions relating to adolescents and analyzing professionals' perceptions regarding the care practices for this age group, through quantitative-qualitative analysis. The results showed that nurses carry out programmed actions such as prenatal appointments, uterine cancer prevention and family planning more often than doctors do. Doctors state that their actions are accomplished during their appointments. Both nurses and doctors say that they provide guidance regarding pregnancy prevention, STD/Aids and condom use. Almost all nurses and just over $50 \%$ of doctors carry out interventional actions. There is a consensus regarding the need to implement a systematized service and for the municipality to capacitate all professionals, restructure the material and human resources, introduce other professionals and integrate the actions with services other than healthcare.

Key words: Adolescent. Family Health Program. Primary prevention. Primary health care.
Entre as diversas atividades desenvolvidas pelos médicos e enfermeiros da Saúde da Família, a presente pesquisa teve como objetivo caracterizar as ações programáticas, preventivas e de intervenção aos adolescentes e analisar a percepção dos profissionais quanto às praticas de atenção a este grupo etário, por meio da análise quanti-qualitativa. Os resultados demonstraram que os enfermeiros realizam as ações programáticas - pré-natal, preventivo do câncer uterino e planejamento familiar com maior frequência do que os médicos. Os médicos referem que seus atendimentos se esgotam na consulta. Ambos profissionais afirmam orientar a prevenção da gravidez, DST/aids e uso do preservativo. Quase a totalidade dos enfermeiros e pouco mais de $50 \%$ dos médicos fazem ações de intervenção. Há consenso quanto à necessidade da implantação de um serviço sistematizado e que o município precisará capacitar todos os profissionais, reestruturar os recursos materiais e humanos, inserir outros profissionais e integrar as ações com serviços, além do da saúde.

Palavras-chave: Adolescente. Programa Saúde da Família. Prevenção. Atenção primaria à saúde.

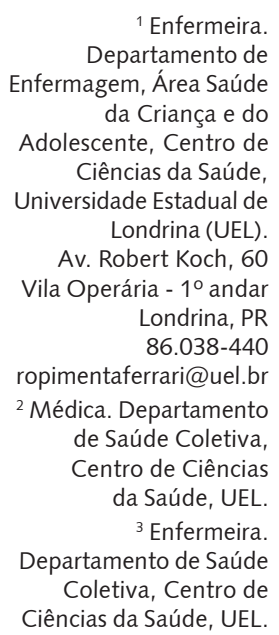




\title{
Introdução
}

No final da década de 1980 e início dos anos 90, o adolescente teve seu reconhecimento como foco de estudo, na sociedade da América Latina e Caribe, no campo da Saúde Pública. Desde então, os direitos e as situações vividas pelos adolescentes resultam, possivelmente, das condições socioeconômicas e das vantagens e desvantagens associadas à classe social, ao gênero e à etnicidade prevalentes na sociedade contemporânea (Cannon, Bottini, 1998).

São essas condições que podem reduzir a possibilidade do acesso equânime dos adolescentes aos serviços, tornando-os mais vulneráveis, dentre os outros grupos etários.

Segundo Alves (1994), o termo vulnerabilidade designa o estado em que se encontram os grupos de indivíduos fragilizados, jurídica e politicamente, quanto à promoção, proteção ou garantia de seus direitos de cidadania.

Neste sentido, o adolescente é considerado vulnerável por ser "um grupo social que se encontra em fase de importantes transformações biológicas e mentais, articuladas a um redimensionamento de identidades e de papéis sociais" (Ayres, França Jr., 1996, p.68).

No entanto, Cannon e Bottini (1998) apontam que, na nossa sociedade, este grupo etário é tratado de forma ambígua, ora como criança, ora como adulto. Ele é capaz de tomar algumas decisões sobre o futuro, todavia deve ser protegido da exploração e do abuso. Recebe informações para saber tomar a decisão correta e comportar-se com prudência nas atitudes concernentes ao bem-estar e à carreira profissional. Logo, é parcialmente independente e dependente, e a aquisição dos direitos adultos ocorre na escala cronológica, muito mais do que pelo seu desenvolvimento físico, mental e social.

Esta ambigüidade de tratamento pode fazer com que os adolescentes demonstrem

\author{
sentimento de invulnerabilidade e, ao mesmo tempo dificuldade de prever as reais \\ conseqüências de seus atos, por acharem que gravidez não planejada, uso de substâncias \\ químicas e contaminação por doenças sexualmente transmissíveis - DST e Aids etc. 'não irão \\ acontecer com eles'. A ocorrência não admitida da possibilidade de danos à própria saúde \\ potencializa-se à medida que não há ações preventivas. (Jeolás, Ferrari, 2003, p.617)
}

Ao reconhecer-se o adolescente como vulnerável, sobretudo no que diz respeito à não-adoção das práticas seguras relacionadas à sexualidade, passa-se a priorizar ações programáticas voltadas para esse segmento populacional.

Desde o final da década de 1980, há iniciativas para se instituírem programas de atenção à saúde do adolescente, como o Programa de Saúde do Adolescente - PROSAD (Brasil, 1989), e estabelecer seus direitos por intermédio do Estatuto da Criança e do Adolescente - ECA (Brasil, 1991). Mas, até então, as políticas públicas para este grupo etário continuam fragmentadas e desarticuladas, mesmo existindo inúmeras iniciativas, tanto governamentais como de grupos organizados da sociedade, o que não tem representado significativamente um trabalho intersetorial para a integralidade da atenção a este grupo etário (Rua, 1998).

$\mathrm{Na}$ atualidade, a Estratégia da Saúde da Família - ESF, considerada um novo modelo de assistência à saúde das populações e de mudanças das práticas profissionais, pode redirecionar as ações programáticas até então instituídas para o grupo de adolescentes, nas diferentes áreas de abrangência dos serviços de atenção básica de saúde.

O profissional de saúde que atua na atenção básica no seu campo de abrangência pode estar mais capacitado para apreensão do quadro de vulnerabilidades locais, inclusive compreender a

[...] dimensão do concreto da vida do adolescente no processo saúde-doença, subsidiando a prática educativa de alcance coletivo em educação, em saúde e dando conta das várias formas de relação dos adolescentes nas esferas da vida nas cidades, da cultura, do trabalho, da instituição educacional, das relações familiares, da sexualidade, do lazer e da Constituição Brasileira. (Peres, Rosenburg, 1998, p. 83) 
Neste cenário, o presente estudo teve como objetivo caracterizar as ações programáticas, preventivas e de intervenção aos adolescentes desenvolvidas pelos médicos e enfermeiros da Saúde da Família, e analisar a percepção dos profissionais quanto às práticas de atenção à saúde para este grupo etário.

\section{Metodologia}

Trata-se de uma investigação descritiva qualitativa e quantitativa, realizada com médicos e enfermeiros das equipes da Saúde da Família do município de Londrina, Paraná, Brasil, no período de agosto a outubro de 2003.

Das 51 Unidades Básicas de Saúde - UBS, cinqüenta possuem equipes da Saúde da Família, totalizando 94 equipes, das quais nove na zona rural e 85 na zona urbana. Cada equipe é composta por nove profissionais: um médico, um enfermeiro, dois auxiliares de enfermagem e cinco agentes comunitários de saúde.

O instrumento de pesquisa contou com questões fechadas de múltipla escolha, que foram analisadas por meio do programa Epi-Info 6.04d (Dean et al., 1995), e uma questão aberta utilizando-se a Análise de Conteúdo, mais especificamente Análise Temática.

Para Bardin (2002, p.105), "o tema é a unidade de significação que se liberta naturalmente de um texto analisado segundo critérios relativos à teoria que serve de guia à leitura". Minayo considera que uma análise temática "consiste em descobrir os núcleos de sentido que compõem uma comunicação cuja presença ou freqüência signifiquem alguma coisa para o objetivo analítico visado" (Minayo, 2001, p. 210).

Para proceder à análise temática, foi organizado e estruturado o conteúdo da questão aberta seguindo as fases seqüenciais propostas por Minayo (2001): a pré-análise, a exploração do material e o tratamento dos resultados.

A pesquisa foi aprovada pelo Comitê de Ética em Pesquisa da Universidade Estadual de Londrina UEL, parecer CEP 176/02, em 11 de dezembro de 2001.

\section{Discussão dos resultados}

Dos 188 profissionais (94 médicos e 94 enfermeiros), 171 participaram da pesquisa, sendo 87,2\% (82) médicos e $94,7 \%$ (89) enfermeiros. Desses, 47 profissionais preencheram a questão aberta do instrumento de pesquisa, que permitiu a realização da análise qualitativa, resultando a categorização de cinco temas extraídos da análise das respostas, a seguir apresentados em três subtópicos quantiqualitativos.

\section{Caracterização do atendimento \& serviços de saúde para adolescentes: pouca oferta ou baixa procura?}

Cerca de 97\% dos médicos e enfermeiros das equipes da Saúde da Família referem que, no serviço de atenção básica de saúde do município, não existe um programa específico para os adolescentes, mas precisam atender outros programas municipal e ministerial.

Quanto ao atendimento do adolescente no serviço, alguns dos registros dos médicos e enfermeiros, submetidos à análise qualitativa, referem-se às ações prestadas a este grupo etário, mas não de forma sistematizada, pois seus atendimentos se esgotam no imediatismo da demanda do serviço: "O atendimento a adolescentes é feito em conjunto à população em geral, dentro da UBS, sem um programa específico" (médico 9); "Não temos programa específico para adolescentes na unidade de saúde, mas procuramos atendê-los da melhor maneira possível com os programas já existentes" (enfermeiro 2).

O atendimento da demanda também esteve presente no estudo qualitativo de Matias (2003) com os médicos das equipes da Saúde da Família de Londrina, no qual referem haver sobrecarga de trabalho para atender a demanda e ser necessário rever esta rotina no serviço de saúde: " [...] precisa ter 
atendimento clínico, só que há de estabelecer uma maneira de você não exaurir a capacidade dos profissionais num atendimento, numa demanda".

Ainda com relação à demanda, estudo de Pedrosa e Teles (2001) com médicos, enfermeiros e Agentes Comunitários de Saúde - ACS das equipes da Saúde da Família, em Teresina/PI, demonstrou que as maiores dificuldades encontradas por eles, foram: o sistema de referência e contra-referência; o aumento da demanda pela chegada de novas famílias; a dificuldade em organizar a demanda do cotidiano da comunidade, que, além das doenças, convivia com violência, drogas, prostituição, problemas para cuja solução os profissionais sentiam-se incapacitados; e, finalmente, a gerência das unidades que representava acúmulo de trabalho.

No serviço de atenção básica do município, os profissionais deste estudo também referem que, dentre a demanda das unidades, priorizam outros programas ministeriais, ao passo que o de atendimento à adolescência está sendo adiado, embora reconheçam a urgência em realizá-lo. Alguns discursos dos profissionais apontam para esta realidade: " [...] estamos trabalhando primeiro com hipertensos e diabéticos da área, depois deste cadastramento e acompanhamento é que vamos partir para um trabalho mais voltado em escolas" (médico 3); "Infelizmente a equipe de Saúde da Família, ainda não se atentou com devida importância às demais áreas além daquelas priorizadas pelas diretrizes do SUS" " (enfermeiro 19); " [...] além dos grupos de hipertensos, visitas dos acamados, a demanda pediátrica é extremamente grande neste serviço, e não consegui ainda organizar o trabalho com adolescentes" (médico 7).

No município, os adolescentes são atendidos de acordo com a procura do serviço. O pediatra atende até os 12 anos de idade, salvo os casos ginecológicos e obstétricos, e, a partir dos 12 anos, são atendidos pelo clínico geral e outras especialidades.

A faixa etária atendida com maior freqüência pelos médicos e enfermeiros é dos 14 aos 16 anos de idade, aproximadamente $50 \%$, e dos 17 aos 19 anos mais de $30 \%$, mas mais de $10 \%$ dos profissionais referem não saber qual a faixa etária que atendiam com maior freqüência.

Com relação ao sexo, os profissionais revelam que a maior procura pelo serviço é do sexo feminino, ao passo que os garotos dificilmente buscam atendimento: "O $\mathrm{n}^{\circ}$ de meninas adolescentes em procura de exames laboratoriais para confirmar gestação tem aumentado... E é uma grande preocupação, pois quando você vai abordar essas meninas é claro sua imaturidade física e emocional, já adolescentes meninos a procura é baixa, mais um ponto para reflexão" (enfermeiro 30 ).

Quanto à freqüência do atendimento, a grande maioria dos médicos $(80,5 \%)$ e pouco mais da metade dos enfermeiros $(51,7 \%)$ atendem o adolescente diariamente ou quase todos os dias.

O fato de os médicos atenderem os adolescentes com maior freqüência do que os enfermeiros pode estar relacionado com a demanda diária das especialidades, como: pediatria, clínica geral, ginecologia e obstetrícia, pois, quando o adolescente procura atendimento no serviço de saúde, geralmente o faz para consulta médica.

Quanto ao motivo da procura dos adolescentes por atendimento médico, mais de $60 \%$ dos médicos afirmam que eles os procuram para tratamento de doenças, e cerca de $40 \%$ por doenças e gravidez.

Tais resultados não diferem dos resultados da pesquisa de Pinheiro et al. (2002), para identificar o acesso e utilização dos serviços de saúde pela população brasileira, e de Formigli, Costa e Porto (2000) sobre o programa de atendimento aos adolescentes em Emaús, Belém-Pará, que mostram que o grupo de adolescentes era o que menos procurava o serviço de saúde - e, quando o procura, geralmente é primeiramente por doença, seguido por gravidez e problemas odontológicos.

Parece que o motivo da procura do adolescente pelo serviço de saúde não tem sido além dos problemas clínicos. Mesmo que o serviço ofereça atividades de prevenção ou de promoção à saúde, alguns dos discursos dos profissionais deste estudo referem que geralmente eles não comparecem, e que ganhar a confiança do adolescente não é uma tarefa fácil: "[...] devido a pouca procura espontânea tendemos a procrastinar as ações" (enfermeiro 3); "Não desenvolvo um programa específico para adolescente por não ter e pela baixa procura dessa faixa de idade ao posto [...]" (médico 6); "[...] a adesão dos adolescentes é pequena, pode ser por falta de experiência nossa" (enfermeiro 6).

Quanto à participação dos jovens em atividades oferecidas no serviço de saúde, os resultados da pesquisa de Jeolás e Ferrari (2003), sobre a opinião dos adolescentes em relação às atividades desenvolvidas, em forma de oficinas, no ambulatório de atendimento espećfíico a este grupo etário, do 
município de Londrina, demonstram que eles consideram um local propício para falar de assuntos dificilmente tratados em outros espaços institucionais. Este espaço também foi considerado por eles como local de prazer, de compartilhamento e aprendizado.

Para Ayres e França Jr., ainda que se proporcione um espaço para este grupo etário, é necessário:

[...] evitar intervalos muito grandes entre o primeiro contato com o serviço e a atividade agendada. Pois um programa para adolescente precisa possuir um agendamento ágil e razoavelmente diversificado. Seus carecimentos Ihes parecem sempre urgentes, seus prazos são sempre... agora. Quanto maior for o intervalo entre a captação e a atividade programática, menor serão as chances de efetivação. (Ayres, França Jr., 1996, p.76)

Segundo Abduch (1999) e Ruzany et al. (2002), os profissionais que operam com adolescentes referem-se às dificuldades encontradas no agendamento de atividades, o que lhes causa frustração, desmotivação, mal-estar e até mesmo irritação, pois os esforços parecem ser em vão. Na verdade, o adolescente:

[...] ainda experimenta integrar a dimensão cronológica de tempo com as nossas práticas [...] tornando-se num processo de aprendizagem [...] exigindo da equipe um alto grau de plasticidade, criatividade e autoridade para que aproveite suas presenças ao máximo, pois não tem a garantia do próximo encontro e da continuidade deste processo. (Abduch, 1999, p. 297)

Para a adesão do adolescente ao espaço que the é oferecido, é necessário permitir que ele seja ouvido, possa expor suas idéias, sentimentos e experiências e, que, também seja respeitado e valorizado. O adolescente não quer nada pronto e, talvez, ele ainda não tenha encontrado este espaço no serviço de saúde (Jeolás, Ferrari, 2003).

$\mathrm{Na}$ realidade, quando os profissionais ganham a confiança dos adolescentes, eles se tornam ouvintes e interlocutores, mas, antes mesmo que aconteça esta interação, é necessário que se efetive uma política pública para ampliar o acesso do adolescente aos serviços de saúde, para criar espaços de discussão e aprofundamento de questões formuladas pelos próprios adolescentes, que sintam prazer em discutir temas dificilmente abordados no cotidiano. O objetivo desse trabalho vai muito além das preocupações higienistas e epidemiológicas (Jeolás, Ferrari, 2003, p.619).

\section{Atividades desenvolvidas pelos médicos e enfermeiros \& ações de atenção à saúde do adolescente}

Com relação às ações programáticas realizadas pelos médicos, pode-se verificar, na Tabela 1 , que pouco mais de $20 \%$ são no pré-natal e preventivo do câncer uterino, e cerca de $32 \%$ no planejamento familiar. Quanto às ações de prevenção, aproximadamente $60 \%$ afirmaram realizar orientações sobre DST/Aids e o uso do preservativo, e, pouco mais da metade, sobre gravidez.

Quanto às ações de prevenção, alguns dos discursos dos médicos referem que, dentre as iniciativas preventivas da equipe, a consulta é um momento oportuno para orientar o adolescente, mesmo que o atenda somente pela procura e a demanda do serviço: "O meu atendimento é feito de acordo com a demanda espontânea. A consulta médica é uma oportunidade de abordar a prevenção primária [...]" (médico 4); " [...] procuro orientá-los durante as consultas sobre a importância de se usar preservativos para evitar a gestação e doenças sexualmente transmissíveis além de recomendar as vacinações" (médico 18); "A prevenção em minha USF é realizada de uma forma individualizada. Existem algumas tentativas por parte da equipe, principalmente dentro da escola, de se organizarem palestras e outras formas de discussão [...]" (médico 16).

A Tabela 2 mostra que, diferentemente dos médicos, cerca de $70 \%$ dos enfermeiros atendem os adolescentes no planejamento familiar, e mais de $70 \%$ no pré-natal e preventivo do câncer uterino. Quanto às ações preventivas, mais de $80 \%$ referem orientar sobre o uso do preservativo, e cerca de $70 \%$ sobre gravidez e DST/Aids. 
Pode-se observar que a diferença do atendimento aos adolescentes, entre as categoriais profissionais, é inerente ao processo de trabalho nas unidades do município, pois mesmo que o médico faça parte da equipe da Saúde da Família, ele cumpre com os números de consultas diárias individuais, preconizados pelo serviço, ao passo que a enfermagem, em suas atividades assistenciais, desenvolve atendimentos grupais no pré-natal e planejamento familiar, consultas puerperais e puericultura, e, também, realiza as campanhas de coleta do preventivo de câncer, vacinação e outros (Londrina, 2006, 2006a).

Além das ações programáticas e preventivas na unidade de saúde, alguns dos discursos dos enfermeiros referem que a equipe tem realizado atividades com os adolescentes na comunidade e na escola: "Há um mês estamos trabalhando junto à ação social e o conselho tutelar na prevenção de violência intrafamiliar e na promoção de atividades de lazer e educação junto a esta população adolescente" (enfermeiro 4); "Estamos montando um grupo de educação sexual na adolescência e prevenção da DST/Aids" (enfermeiro 13); "Estamos fazendo trabalho na escola, pois achamos que atingimos melhores resultados com os adolescentes" (enfermeiro 18).

Com relação às atividades de intervenção, pode-se observar, nas Tabela 1 e 2, que pouco mais de $50 \%$ dos médicos e quase a totalidade dos enfermeiros indica e/ou aplica a vacinação para os adolescentes.

Tabela 1. Distribuição dos médicos das equipes da Saúde da Família, segundo as atividades desenvolvidas no atendimento aos adolescentes, Londrina, 2003.

\begin{tabular}{|c|c|c|c|c|c|c|}
\hline \multirow[t]{2}{*}{ Atividades desenvolvidas } & \multicolumn{2}{|c|}{ Realizavam } & \multicolumn{2}{|c|}{ Não realizavam } & \multicolumn{2}{|c|}{ Total } \\
\hline & $\mathrm{N}^{\circ}$ & $\%$ & $\mathrm{~N}^{\circ}$ & $\%$ & $\mathrm{~N}^{\circ}$ & $\%$ \\
\hline \multicolumn{7}{|l|}{ Ações Programáticas } \\
\hline Planejamento familiar & 26 & 31,7 & 56 & 68,3 & 82 & 100,0 \\
\hline Pré-natal & 18 & 22,0 & 64 & 78,0 & 82 & 100,0 \\
\hline Preventivo ca uterino & 20 & 24,4 & 62 & 75,6 & 82 & 100,0 \\
\hline \multicolumn{7}{|l|}{ Ações Preventivas } \\
\hline DST e Aids & 50 & 61,0 & 32 & 39,0 & 82 & 100,0 \\
\hline Gravidez & 44 & 53,6 & 38 & 46,3 & 82 & 100,0 * \\
\hline Uso do preservativo & 49 & 59,8 & 33 & 40,2 & 82 & 100,0 \\
\hline \multicolumn{7}{|l|}{ Intervenções } \\
\hline Vacinação: indicação e/ou aplicação & 45 & 54,9 & 37 & 45,1 & 82 & 100,0 \\
\hline Curativos: indicação e/ou aplicação & 21 & 25,6 & 61 & 74,4 & 82 & 100,0 \\
\hline
\end{tabular}

* Aproximado para $100 \%$

Tabela 2. Distribuição dos enfermeiros das equipes da Saúde da Família, segundo as atividades desenvolvidas no atendimento aos adolescentes, Londrina, 2003.

\begin{tabular}{|c|c|c|c|c|c|c|}
\hline \multirow[t]{2}{*}{ Atividades desenvolvidas } & \multicolumn{2}{|c|}{ Realizavam } & \multicolumn{2}{|c|}{ Não realizavam } & \multicolumn{2}{|c|}{ Total } \\
\hline & $\mathrm{N}^{\circ}$ & $\%$ & $\mathrm{~N}^{\circ}$ & $\%$ & $\mathbf{N}^{\circ}$ & $\%$ \\
\hline \multicolumn{7}{|l|}{ Ações Programáticas } \\
\hline Planejamento familiar & 77 & 86,5 & 12 & 13,5 & 89 & 100,0 \\
\hline Pré-natal & 68 & 76,4 & 21 & 23,6 & 89 & 100,0 \\
\hline Preventivo ca uterino & 66 & 74,1 & 23 & 25,8 & 89 & 100,0 \\
\hline \multicolumn{7}{|l|}{ Ações Preventivas } \\
\hline DST e Aids & 62 & 69,7 & 27 & 30,3 & 89 & 100,0 \\
\hline Gravidez & 63 & 70,8 & 26 & 29,2 & 89 & $100,0 *$ \\
\hline Uso do preservativo & 73 & 82,0 & 16 & 18,0 & 89 & 100,0 \\
\hline \multicolumn{7}{|l|}{ Intervençõ̃es } \\
\hline Vacinação: indicação e/ou aplicação & 86 & 96,6 & 3 & 3,4 & 89 & 100,0 \\
\hline Curativos: indicação e/ou aplicação & 79 & 88,8 & 10 & 11,2 & 89 & 100,0 \\
\hline
\end{tabular}

* Aproximado para $100 \%$ 
Evidencia-se que a maior atuação dos enfermeiros em procedimentos é proveniente da própria rotina de trabalho da enfermagem, pois trata de atendimentos inerentes à sua função.

Quanto ao pré-natal, a pouca participação dos médicos, deste estudo, pode ser devida ao fato de que a maior parte dos especialistas em ginecologia e obstetrícia não esteja vinculada à Estratégia da Saúde da Família - ESF e, conseqüentemente, esse procedimento seja realizado dentro da rotina de especialidades do serviço.

Pesquisas para identificar os fatores associados à inadequação do uso de assistência pré-natal em comunidade urbana, nas maternidades do Município de São Luís/MA (Coimbra et al., 2003), e analisar a situação da atenção ao pré-natal oferecida pela equipe da Saúde da Família, em quatro microrregiões de saúde de Baturité, Ceará (Moura, Holanda Jr., Rodrigues, 2003), apontam que, em geral, apesar de a cobertura da ESF ser de quase $100 \%$, a mulher adolescente ainda pertencia a um segmento populacional com deficiente uso da assistência pré-natal.

Quanto às ações programáticas (planejamento familiar, pré-natal e câncer ginecológico) e preventivas (DST/Aids, gravidez e uso do preservativo), Costa Neto (2000) refere que tanto os médicos como os enfermeiros têm competência e habilidade para desenvolvê-las, tanto por intermédio de consultas na unidade, como em reuniões com grupos e em visitas domiciliares. Indiferentemente do local para desenvolver estas atividades, deve-se levar em consideração que, em meio à demanda da unidade, este constitui-se num espaço educativo muito rico e, ao mesmo tempo, muito curto. $\mathrm{O}$ profissional tem de estar preparado para bem aproveitá-lo. Na prática, parece que aproveitar este espaço não tem sido uma tarefa tão fácil, ainda mais com enfoque na prevenção, é o que refere um dos discursos dos profissionais: " [...] Lidar com as questões que envolvem os adolescentes é um grande desafio para os profissionais de saúde hoje" (enfermeiro 22).

Neste estudo, pode-se observar que grande parte dos médicos e enfermeiros afirma realizar a prevenção das DST/Aids, gravidez e orientação do uso do preservativo. Entretanto, ainda há que se avaliar a efetividade das ações realizadas por estes profissionais.

A efetividade parece ser uma das metas das equipes de saúde, mas foge da governabilidade das ações, como a gravidez na adolescência; e alguns dos discursos dos profissionais deste estudo apontam para o crescente aumento nas áreas de abrangência, mas ainda as intervenções preventivas têm sido insuficientes, pois o acesso deste grupo etário se limita na procura espontânea, e quando a faz, geralmente a adolescente está grávida: " [...] em nossa área de abrangência as adolescentes têm iniciado vida sexual, digo é grande o $\mathrm{n}^{\circ}$ de adolescentes, e o $\mathrm{n}^{\circ}$ de gravidez nessa faixa etária é significante" (enfermeiro 12); "Temos um aumento no $\mathrm{n}^{\circ}$ de meninas que vem engravidando, e ainda não conseguimos elaborar uma estratégia na busca de acabar com esse problema" (enfermeiro 15); "Na verdade a procura da UBS pelo adolescente ainda é pouco, e em nosso distrito estamos com alto índice de gravidez na adolescência [...]" (enfermeiro 21).

Ainda que a gravidez na adolescência aparentemente não esteja sob a governabilidade do serviço, alguns discursos sugerem a necessidade do desenvolvimento de ações preventivas, e que a abordagem deverá ir além dos modelos tradicionais, para, então, mudar o cenário atual dos índices de gravidez e da transmissão das DST/Aids: "[...] Quanto à saúde poderia haver um maior incentivo à prevenção de gestação indesejada e DST" (médico 5); "O enfoque a ser dado na prevenção de DST para adolescente e prevenção de gravidez precoce não deveria ser apenas o uso de anticoncepcionais, preservativos, assim como a campanha para diminuir o abuso de drogas e a transmissão de doenças não se baseia apenas na distribuição de seringas descartáveis. É necessário uma orientação da sexualidade no sentido total e não apenas genital. Paulatinamente com um trabalho também com a família (apesar da realidade complicada de alguns lugares) [...]" (médico 10); "A educação é a principal forma de prevenção de gravidez precoce e DST, necessitando uma melhor orientação dos professores para que possam abordar o tema da sexualidade de forma natural e instrutiva [...]" (médico 14).

A qualidade do desenvolvimento das ações de prevenção no serviço de atenção básica é tema de estudo entre vários pesquisadores; e os resultados não têm sido positivos, visto que ainda é crescente, entre os adolescentes, a menor idade da iniciação sexual, a gravidez, o aborto, as DST e a Aids (Paiva et al., 2003; Brasil, 2002; Huertas, 2001; Camacho Hubner, 2000; Santos, Santos, 1999; Alan Guttmacher Institute, 1998; Ayres, Calazans, França Jr., 1998). 
A pesquisa de Torres (2000) para identificar a efetividade das atividades desenvolvidas pelos enfermeiros no programa de DST/Aids, nas UBS do Município de Natal/RN, conclui que mais de $90 \%$ dos enfermeiros desenvolviam ações de prevenção quanto ao uso do preservativo; entretanto, as orientações individuais eram feitas esporadicamente e exclusivamente para a demanda espontânea, ou seja, não existia programação específica com metas, objetivos e estratégias preventivas. E o estudo de Moura e Sousa (2002), para levantar as causas da baixa oferta de ações educativas em saúde reprodutiva, realizado pelas equipes da Saúde da Família, nas UBS da sede dos oito Municípios da microrregião de Baturité, no Estado do Ceará, demonstrou que as atividades em educação em saúde desenvolvidas pelos profissionais eram verticalizadas, com temas predefinidos, permitindo pouca ou nenhuma interação com a clientela; que a orientação era individual, oferecida por ocasião das consultas, restringia-se ao simples repasse de informações, levando à conclusão de que esta prática é proveniente da falta de conhecimento e habilidade dos profissionais quanto à realização adequada dessas atividades.

Segundo Paiva (1994), a baixa eficácia de muitas ações preventivas nos serviços de saúde se deve a erros já globalmente identificados em muitos países de terceiro mundo, referentes às decisões sobre políticas públicas de prevenção que não têm levado em conta a cultura sexual, especialmente o contexto social e cultural em que as decisões sobre sexo são tomadas, não indo muito além das informações genéricas sobre gravidez, DST e Aids.

Enquanto as ações de promoção e de prevenção continuam aquém do desejado, mesmo com as mudanças das políticas de saúde e das práticas profissionais nos serviços de atenção básica de saúde, pesquisas demonstram que um dos maiores desafios dos serviços de saúde não só no Brasil, mas também em todo o mundo, é manter o uso consistente do preservativo entre os adolescentes, que, anualmente, têm iniciado precocemente vida sexual e sem proteção, com significativa relação entre a maior fecundidade e a baixa escolaridade e, conseqüentemente, reduzir os índices da gravidez não planejada, das DST e da Aids (Brasil, 2004; Paiva et al., 2003; Simões et al., 2003; Gama, Szwarcwald, Leal, 2002; Lunardelli, 2002; Camacho Hubner, 2000; Sociedade Civil Bem-Estar Familiar no Brasil, 1999; Ferraz, Ferreira, 1998).

No município de Londrina, a média da iniciação sexual é de 14,2 anos para os meninos e 15,1 para as meninas (Oliveira, 2002), e nos países da América Latina e Caribe, de 15,7 anos para homens e 17,0 anos para mulheres (Huertas, 2001).

Se há precocidade da iniciação sexual, o adolescente é mais propenso a dispensar o preservativo, pela restrição do acesso ou por não ser capaz de convencer o parceiro ou parceira da necessidade do seu uso, especialmente o grupo feminino, tornando-se o mais vulnerável, entre outras causas. A relação sexual sem o preservativo, na presença de uma DST, aumenta o risco de transmissão da Aids de três a cinco vezes (Brasil, 2003, 2002). Anualmente, um entre vinte adolescentes contrai algum tipo de DST e, diariamente, mais de sete mil jovens - cinco por minuto - são infectados pelo HIV, num total de 2,6 milhões por ano, o que representa a metade de todos os casos registrados. Estima-se que dez milhões de adolescentes vivam hoje com o HIV ou estão propensos a desenvolver a Aids no período dos próximos três a 15 anos. Aproximadamente $80 \%$ das transmissões do HIV ocorrem por sexo desprotegido (Brasil, 2002).

Esta realidade reafirma a necessidade da efetivação de uma política pública para ampliar o acesso dos adolescentes ao serviço de saúde, e da mudança urgente das práticas dos profissionais da Saúde da Família, pois os modelos tradicionais da atenção médica e de saúde pública são fragmentados e desintegrados e não responderão às necessidades dos adolescentes; ao passo que o modelo de atenção sob a ESF, que consta na Constituição Federal, nas Constituições e Leis Orgânicas Municipais e na Legislação do SUS, destina-se a cumprir os princípios da integralidade, priorizando as ações preventivas e promocionais, a universalização da assistência nos níveis de atenção da promoção, proteção, recuperação e reabilitação.

Estas ações, embasadas pelas noções de territorialização, integralidade da atenção e impacto epidemiológico, reorientando o planejamento de saúde para uma base populacional específica, como o adolescente, gerará a promoção da saúde com medidas gerais e a proteção com medidas específicas para a prevenção da ocorrência da gravidez não planejada, das DST e da Aids. 


\section{Adolescência - necessidades \& complexidades para implantação de um programa}

Na Figura 1, evidencia-se que mais de $80 \%$ dos profissionais consideram ser prioritário atender o adolescente no serviço de saúde, no entanto apenas 32,5\% dos médicos e 41,2\% dos enfermeiros referem desenvolver ações de atenção à saúde do adolescente.

Mesmo que os profissionais afirmem que não há um programa específico de atendimento a este grupo etário no serviço de saúde, alguns dos discursos dos profissionais evidenciam a predisposição e necessidade de se implantar um programa de atenção à saúde do adolescente no serviço: "Quanto às ações à saúde do adolescente, é prioritário, de suma importância, necessário e urgente [...] (enfermeiro 2); "Acredito que deveria ter um programa mais específico para o atendimento aos adolescentes nas UBS [...]" (médico 19); "Acho que a Autarquia Municipal de Saúde precisa implantar um programa de atenção ao adolescente [...]" (enfermeiro 7).

Quanto à viabilidade da implantação de um programa para adolescentes na unidade de saúde, outros discursos apontam para a dificuldade em abordá-los, pela escassa capacitação no serviço, barreiras estruturais e familiares, entre outros: " [...] nas UBS há muito pouco desenvolvimento, pois quase não temos embasamento quanto a este programa [...]" (enfermeiro 2); “ [...] estamos encontrando dificuldades na abordagem dos adolescentes, aceitação dos pais em relação a alguns assuntos, principalmente sobre sexo" (enfermeiro 21).

Antes da execução de um programa para este grupo populacional no serviço de saúde, alguns dos discursos dos médicos e enfermeiros apontam para a necessidade de formação/capacitação sobre a temática, pois no serviço há treinamentos periódicos, mas nenhum em adolescência: “[...] não temos um treinamento maior para atender a essa população" (médico 4); " [...] Gostaria de ter qualificação melhor para atendê-los, saber como abordar os assuntos que fazem parte dessa fase" (enfermeiro 5); " [...] Os funcionários devem ser treinados para trabalhar neste programa" (enfermeiro 7); "Como nós médicos e enfermeiros do PSF de Londrina recebemos periodicamente treinamentos, através do programa de educação continuada da prefeitura, e ainda não tivemos nenhum tipo de treinamento em relação a adolescentes, a minha sugestão é que esse tema possa ser abordado futuramente" (médico 11).

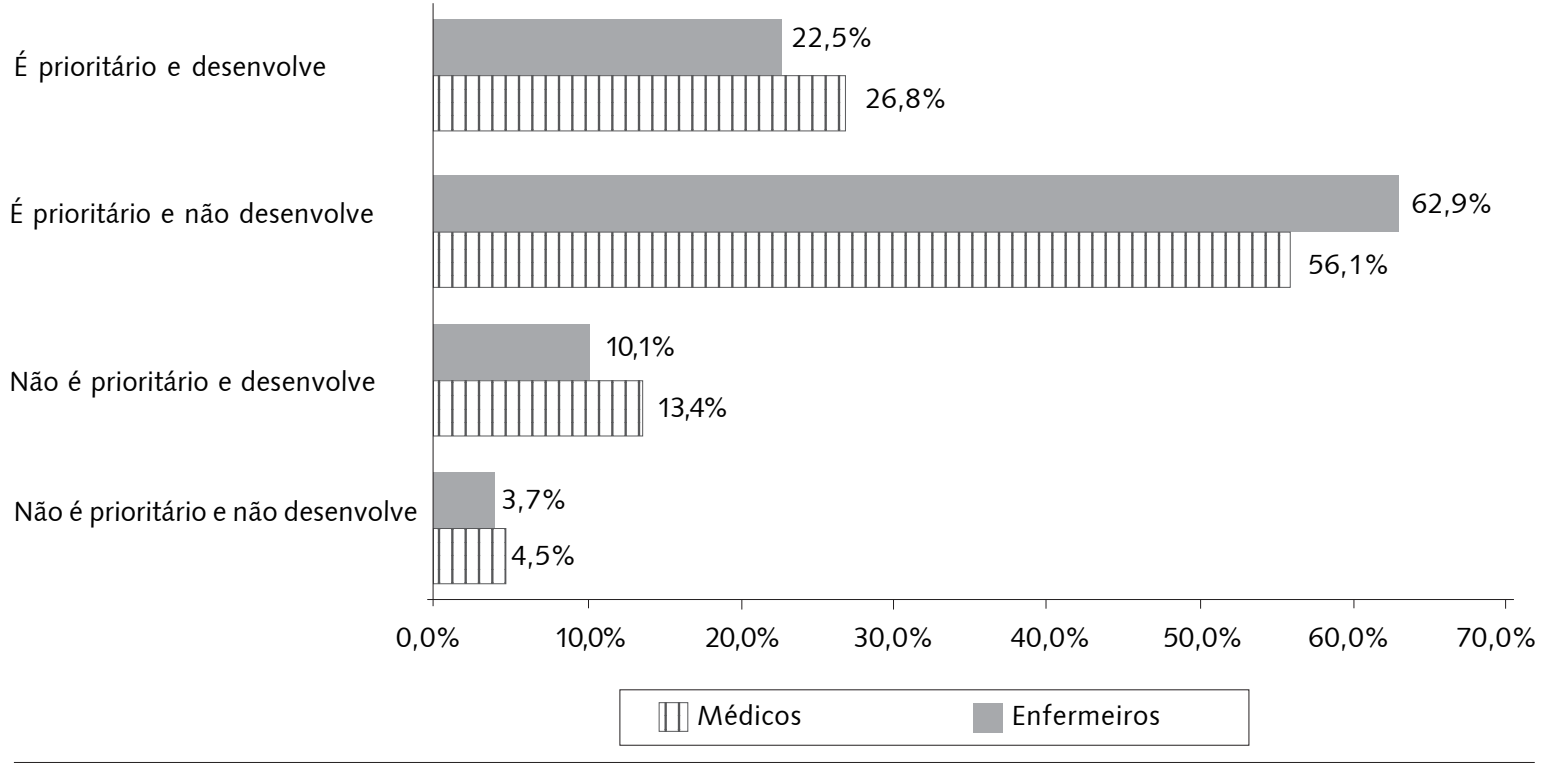

Figura 1. Distribuição dos médicos e dos enfermeiros das equipes da Saúde da Família, segundo a opinião sobre a prioridade do atendimento ao adolescente no serviço de saúde, Londrina, 2003. 
Para desenvolver atividades num programa para adolescente, exige-se um enfoque mais amplo, não apenas nos aspectos técnico e biológico, mas também nos aspectos psicossociais, históricos, sociais, culturais, políticos, nos valores e comportamentos - e nem sempre os profissionais se sentem aptos para atuar nesta complexidade de saberes.

Segundo Aguiar, Bock e Ozella (2001, p.171), "é preciso superar visões naturalizantes e entender a adolescência como constituída socialmente a partir das necessidades sociais e econômicas dos grupos sociais e olhar e compreender suas características como características que vão se constituindo no processo".

A partir deste processo, a adolescência concebida historicamente "determinará as ações pessoais, políticas, sociais, profissionais em relação a ela" (Ozela et al., 2003, p.9).

Sendo assim, nesta proposta de programa, o domínio clínico não prevalecerá, mas por meio do modelo de atenção integral, sob a ESF, poderá viabilizar outras possibilidades de atenção à saúde, pois, para discutir prevenção e sexo seguro, é necessário falar sobre os aspectos que permeiam a sexualidade humana na nossa sociedade.

Esta abordagem não possui caráter ou modelos definitivos, e a concepção pessoal se torna dispensável; e os profissionais não poderão ser transmissores de conhecimento de cima para baixo, nem tampouco o de valores pessoais relativos à sexualidade, numa atitude disciplinar empenhada em manter o status quo sexual e da sacralidade da ordem social estabelecida. Ele deverá ser o de interlocutor e de facilitador da discussão e da reflexão. (Jeolás, Ferrari, 2003, p.618)

Além da mudança das práticas profissionais, para obter efetividade nas intervenções junto ao adolescente, será necessária também " [...] a construção de novos modelos de organização de trabalho em saúde que possibilite avaliação retrospectiva das ações desenvolvidas pela equipe multidisciplinar e um planejamento prospectivo de sua aplicação" (Ayres, 1990, p.176).

Quanto ao trabalho multidisciplinar, alguns dos discursos dos médicos e enfermeiros referem esta necessidade, mas a realidade do serviço parece ser outra: "[...] a adolescência envolve um período de transformação psicológica muito importante, o que contribui para a grande incidência de gravidez precoce e DST. Logo, minha opinião é que deveríamos priorizar a educação do adolescente em nível psicológico, uma vez que o planejamento familiar e a prevenção de DST já vem sendo realizada, mas esbarra na falta de preparo emocional para esta fase da vida" (médico 2); "[...] sempre há necessidade de uma psicóloga, porém é um profissional que não temos" (enfermeiro 6); "O atendimento ao adolescente com qualidade e preparo dos diferentes profissionais não existe na rede pública municipal" (médico 12).

A adolescência nas ciências médicas e humanas é uma temática multidisciplinar e interdisciplinar, pois consiste numa modalidade de trabalho coletivo que se configura na relação recíproca entre as múltiplas intervenções técnicas e a interação dos agentes de diferentes áreas profissionais (Peduzzi, 2001). Um dos discursos aponta para esta necessidade: "Trabalhos com adolescentes devem ser multidisciplinares, principalmente participação de psicólogo ou pediatra com especialidade em lidar com adolescentes" (médico 1).

Cabe salientar que a necessidade de um psicólogo no trato com o adolescente pode ser factível, mas não é uma realidade das equipes da Saúde da Família; no entanto os profissionais que atuam na estratégia podem atender as maiores necessidades deste grupo etário, desde que estejam capacitados e haja reorganização do processo de trabalho nas unidades de saúde.

Neste sentido, Bertussi, Oliveira e Lima (2001, p.142) referem que o trabalho multiprofissional não requer:

[...] vários profissionais atuando num mesmo serviço, mas sim um grupo de profissionais conhecedor da missão do serviço de saúde, compreendendo sua tarefa, e atingindo os objetivos e metas estabelecidas no planejamento estratégico para a sua área de atuação, o qual deve ser elaborado de forma participativa e democrática. 
O trabalho realizado em equipe, com interação e troca nos campos de competência de cuidado, abre a possibilidade de cada um usar todo o seu potencial criativo na relação com o usuário, para juntos realizarem a produção do cuidado (Franco, Merhy, 1999).

Para Ayres (1990, p.144) "[...] não se trata de somar conhecimentos, mas transformá-los num outro, apropriado às necessidades específicas do trabalho com adolescentes".

Além da multidisciplinariedade no trato com o adolescente, os profissionais precisam buscar parcerias com outros setores a fim de obter melhor e maior efetividade nas ações de atenção integral à saúde deste grupo etário. Alguns dos discursos dos médicos e enfermeiros revelam a dificuldade em utilizar o serviço de referência e contra-referência e em promover a intersetorialidade: " [...] dificuldade de encaminhar adolescentes com problemas para profissionais e serviços específicos" (enfermeiro 8); "Para o atendimento ser mais efetivo deveria existir uma parceria entre a Secretaria de Saúde e Secretaria de Educação" (enfermeiro 24); "Acho que principalmente o PSF poderia realizar parcerias com as escolas para o desenvolvimento de ações à saúde dos adolescentes. Poderíamos abordar assuntos como drogas, planejamento familiar, auto-estima e etc. Porém isso só seria possível através de parcerias bem engajadas com a Secretaria de Saúde e Educação, pois até procedimentos simples, como a vacinação nas escolas, encontramos algumas dificuldades com a Secretaria de Educação" (enfermeiro 27).

Um dos desafios da intersetorialidade é identificar objetivos comuns e, mediante um núcleo estratégico de planejamento e definição, buscar prioridades de ações em conjunto. Para tanto, Raynaut (2002, p.44) alerta que "[...] a complexidade do real não pode ser abordada a partir de apenas um ângulo ou de um nível de análise, é necessário juntar os olhares e as competências".

\section{Considerações finais}

Cabe ressaltar que este estudo teve por objetivo fazer uma análise descritiva das ações desenvolvidas pelos médicos e enfermeiros para os adolescentes, e analisar a percepção quanto à execução destas atividades no serviço de atenção básica de saúde no município.

Certamente, a pesquisa poderá contribuir para o aprofundamento da temática adolescência e a Estratégia da Saúde da Família, com a realização de novas pesquisas utilizando outras metodologias, e, também para o estabelecimento e adequação de intervenções e/ou readequações das políticas públicas existentes a quase duas décadas para os adolescentes (PROSAD e ECA), numa perspectiva de atenção integral, intersetorial e equânime.

\section{Agradecimentos}

Primeiramente, aos médicos e enfermeiros das equipes da Saúde da Família do município, pois sem a participação de cada um a pesquisa não teria sido viabilizada. Em especial, à Dra. Leila Sollberger Jeolás, antropóloga docente do Centro de Ciências Humanas da Universidade Estadual de Londrina, pelas contribuições e momentos reflexivos na temática. Às estudantes do Curso de Enfermagem da Universidade Estadual de Londrina, Bruna Camila F. da Silva, Fernanda Donádio Pitta e Giseli Plath, pela participação na pesquisa.

\section{Colaboradores}

Os autores Rosângela Aparecida Pimenta Ferrari, Zuleika Thomson e Regina Melchior participaram, igualmente, de todas as etapas de elaboração do artigo. 


\section{Referências}

ABDUCH, C. Grupos operativos com adolescentes. In: SCHOR, N.; MOTA, M. S.F.T.; BRANCO, V.C. (Orgs.). Cadernos juventude, saúde e desenvolvimento. Brasília: Ministério da Saúde, 1999. p.289-300.

AGUIAR, W.M.J.; BOCK, A.M.B; OZELLA, S.A. A orientação profissional com adolescentes: um exemplo de prática na abordagem sócio-histórica. IN: BOCK, A.M.B.; GONÇALVES, M.G.M.; FURTADO, O. (Orgs.). Psicologia sócio-histórica: uma perspectiva crítica em psicologia. São Paulo: Cortez, 2001. p.163-78.

ALVES, J.A.L. Os direitos humanos como tema global. São Paulo: Perspectiva, 1994.

ALAN GUTTMACHER INSTITUTE. Into a new world: young women's sexual and reproductive lives. New York: CEPIA, 1998.

AYRES, J.R.C.M. Adolescência e saúde coletiva: aspectos epistemológicos da abordagem programática. In: SCHRAIBER, L.B. (Org.). Programação em saúde hoje. São Paulo: Hucitec, 1990. p.139-82.

AYRES, J.R.C.M.; CALAZANS, G.J.; FRANÇA JR., I. Vulnerabilidade do adolescente ao HIV/ Aids. In: VIEIRA, E.M. et al. (Orgs.). Seminário gravidez na adolescência. Rio de Janeiro: Associação Saúde da Família, 1998. p.97-109.

AYRES, J.R.C.M.; FRANÇA JR., I. Saúde do adolescente. In: SCHRAIBER, L.B.; NEMES, M.I.B.; GONÇALVES, R.B.M. (Orgs.). Saúde do adulto: programa e ações na unidade básica. São Paulo: Hucitec, 1996. p.67-85.

BARDIN, L. Análise de conteúdo. Lisboa: Edições 70, 2002.

BERTUSSI, D.C.; OLIVEIRA, M.S.M.; LIMA, J.V.C. A unidade básica no contexto do sistema de saúde. In: ANDRADE, S.M.; SOARES, D.A.; CORDONI JR., L. (Orgs.). Bases da saúde coletiva. Londrina: UEL, 2001. p.133-43.

BRASIL. Ministério da Justiça. Departamento da Criança e do Adolescente. Estatuto da Criança e do Adolescente; Brasília, 1991.

BRASIL. Ministério da Saúde. Programa Saúde da Mulher. Brasília, 2004. Disponível em: <http://www.saúde.gov.br/sps/htm>. Acesso em: 10 jan. 2006.

Boletim epidemiológico: Aids. Brasília, 2002. Disponível em:

<http://www.aids.gov.br/htm>. Acesso em: 20 jul. 2006.

Secretaria de Políticas de Saúde. Programa Nacional de DST e Aids. Dados e pesquisas em DST e Aids. Brasília, 2003. Disponível em: <http://www.aids.gov.br/final/ dados/dst.htm>. Acesso em: 09 jun. 2006.

Secretaria Nacional de Programas Especiais de Saúde. Divisão Nacional de Saúde Materno-Infantil. Programa de Saúde do Adolescente: bases programáticas. Brasília, 1989.

CAMACHO HUBNER, A.V. Perfil de salud sexual y reprodutiva de los y las adolescentes y jovens de América Latina y el Caribe: revisión bibliográfica, 1988-1998. Washington: OPS, 2000

CANNON, L.R.C.; BOTTINI, B.A. Saúde e juventude: o cenário das políticas públicas no Brasil. In: BRASIL. Ministério do Planejamento e Orçamento. Jovens acontecendo na trilha das políticas públicas. Brasília: CNPD, 1998. p.397-416. v.1.

COIMBRA, L.C. et al. Fatores associados à inadequação do uso da assistência pré-natal. Rev. Saúde Pública, v.37, n.4, p.456-62, 2003. Disponível em: <http://www.scielo.br/ html>. Acesso em: 26 jan. 2006.

COSTA NETO, M.M. (Org.). Competências para o trabalho em uma unidade básica de saúde sob a estratégia de saúde da família: médico e enfermeiro. Brasília: Ministério da Saúde, 2000.

DEAN, A.G. et al. Epi Info, version 6.04d a Word processing, database and statistics program for epidemiology on microcomputers. Atlanta: Centers for Disease Control and Prevention, 1995. 
FERRAZ, E.; FERREIRA, I.Q. Início da atividade sexual e características da população adolescente que engravida. In: VIEIRA, E.M. et al. (Orgs.). Seminário gravidez na adolescência. Rio de Janeiro: Associação Saúde da Família, 1998. p.47-54.

FORMIGLI, V.L.A.; COSTA, M.C.O.; PORTO, L.A. Avaliação de um serviço de atenção integral à saúde do adolescente. Cad. Saúde Pública, v.3, n.16, p.831-41, 2000.

FRANCO, T.B.; MERHY, E.E. PSF: contradições de um programa destinado à mudança do modelo tecnoassistencial. Campinas, 1999.

GAMA, S.G.N.; SZWARCWALD, C.L.; LEAL, M.C. Experiência de gravidez na adolescência, fatores associados e resultados perinatais entre puérperas de baixa renda. Cad. Saúde Pública, v.18, n.1, p.153-61, 2002. Disponível em: <http://www.scielo.br/ html>. Acesso em: 26 jan. 2004.

HUERTAS, L.L.R. Reforma sanitária, promocion de la salud y programas de salud de las adolescentes en la region: casos de Brasil, Costa Rica y República Dominicana. Washington: OPS, 2001.

JEOLÁS, L.S.; FERRARI, R.A.P. Oficinas de prevenção em um serviço de saúde para adolescentes: espaço de reflexão e de conhecimento compartilhado. Cienc. Saúde Coletiva, v.8, n.2, p.611-620, 2003.

LONDRINA. Saúde da criança: protocolo. Londrina: Prefeitura/Autarquia Municipal de Saúde, 2006.

Saúde da mulher: protocolo Londrina: Prefeitura/Autarquia Municipal de Saúde, 2006a.

LUNARDELLI, J.L. Anticoncepção na adolescência. Pediatr. Mod., v.38, n.8, p.381-7, 2002.

MATIAS, M.C.M. Trabalho, educação e identidade: problematizando a formação e a prática do médico no Programa da Saúde da Família. 2003. Tese (Doutorado em Educação Médica) - Faculdade de Educação, Universidade de Campinas, Campinas. 2003.

MINAYO, M. C.S. Ciência, técnica e arte: o desafio da pesquisa social. In: Pesquisa social: teoria, método e criatividade. 18.ed. Petrópolis: Vozes, 2001 . p. 9-29.

MOURA, E.R.; HOLANDA JR., F.; RODRIGUES, M.S. Avaliação da assistência pré-natal oferecida em uma microrregião de saúde do Ceará, Brasil. Cad. Saúde Pública, v.19, n.6, p.1791-9, 2003. Disponível em: <http://www.scielo.br/html>. Acesso em: 26 nov. 2005.

MOURA, E.R.F.; SOUSA, R.A. Educação em saúde reprodutiva: proposta ou realidade do Programa Saúde da Família?. Cad. Saúde Pública, v.18, n.6, p.1809-11, 2002. Disponível em: <http://www.scielo.br/html>. Acesso em: 26 jan. 2006.

OLIVEIRA, R.L.C. Estudo sobre o conhecimento de doenças sexualmente transmissíveis e comportamento sexual de adolescentes do ensino médio de escolas públicas de Londrina, PR. 2002. Dissertação (Mestrado em Saúde Coletiva) Universidade Estadual de Londrina, Londrina. 2002.

OZELLA, S. (Org.). Adolescências construídas: a visão da psicologia sócio-histórica. São Paulo: Cortez, 2003.

PAIVA, V. Sexualidade e gênero num trabalho com adolescentes para prevenção do HIV/Aids. In: PARKER, A. et al. (Orgs.). A Aids no Brasil (1982-1992). Rio de Janeiro: $A B I A, 1994$. p. 231-50.

PAIVA, V. et al. Uso de preservativos: pesquisa nacional MS/Ibope, Brasil. 2003. Disponível em:<http://www.aids.gov.br/htm>. Acesso em: 20 dez. 2005.

PEDROSA, J.I.S.; TELES, J.B.M. Consenso e diferenças em equipes do programa saúde da família. Rev. Saúde Pública, v.35, n.3, 2001. Disponível em:

<http://www.scielo.br/html>. Acesso em: 26 fev. 2004. 
PEDUZZI, M. Equipe multiprofissional de saúde: conceito e tipologia. Rev. Saúde Pública, v.35, n.1, p.103-9, 2001. Disponível em: <http://www.scielo.br/html>. Acesso em: 26 jan. 2005.

PERES, F.; ROSENBURG, C.P. Desvelando a concepção de adolescência/ adolescente presente no discurso da Saúde Pública. Saúde Soc., v.7, n.1, p.53-86, 1998.

PINHEIRO, R.S. et al. Gênero, morbidade, acesso e utilização de serviços de saúde no Brasil. Cienc. Saúde Coletiva, v.7, n.4, p.687-707, 2002.

RAYNAUT, C. Interdisciplinaridade e promoção da saúde: o papel da antropologia. Rev. Bras. Epidemiol., v.5, supl.1, p.43-55, 2002.

RUA, M.G. As políticas públicas e a juventude dos anos 90, 1998. In: COMISSÃO NACIONAL DE POPULAÇÃO E DESENVOLVIMENTO. Jovens acontecendo na trilha das políticas públicas. Brasília: Ministério da Saúde/Secretaria Executiva/CNPD, 1998. v.2. p.731-49.

RUZANY, M.H. et al. Avaliação das condições de atendimento do Programa de Saúde do Adolescente no município do Rio de Janeiro. Cad. Saúde Pública, v.18, n.3, p.639-49, 2002.

SANTOS, V.L.; SANTOS, C.E. Adolescentes, jovens e aids no Brasil. In: SCHOR, N.; MOTA, M.S.F.T.; BRANCO, V.C. (Orgs.). Cadernos juventude, saúde e desenvolvimento. Brasília: Ministério da Saúde, 1999. v.1. p.213-22.

SIMÕES, V.M.F. et al. Características da gravidez na adolescência em São Luiz, Maranhão. Rev. Saúde Pública, v.37, n.5, p.559-65, 2003. Disponível em: <http://www.scielo.br/htm>. Acesso em: 23 jan. 2004.

SOCIEDADE CIVIL BEM-ESTAR FAMILIAR NO BRASIL. Adolescentes, jovens e a pesquisa nacional sobre demografia e saúde: um estudo sobre fecundidade, comportamento sexual e saúde reprodutiva. Rio de Janeiro: CDC/Unicef,1999.

TORRES, G. V. Atividades do enfermeiro na prevenção do HIV/Aids em Natal/RN: competências pedagógica e técnica. 2000. Tese (Doutorado em Enfermagem) - Escola de Enfermagem de Ribeirão Preto, Universidade de São Paulo, Ribeirão Preto. 2000.

FERRARI, R.A.P.; THOMSON, Z.; MELCHIOR, R. Adolescencia: acciones y percepción de los médicos y enfermeros del Programa Salud de la Familia. Interface - Comunic., Saúde, Educ., v.12, n.25, p.387-400, abr./jun. 2008.

Entre las diversas actividades desarrolladas por los médicos y enfermeros de la Salud de la Familia, el objetivo de la presente pesquisa ha sido caracterizar las acciones programáticas, preventivas y de intervención a los adolescentes y analizar la percepción de los profesionales en cuanto a las prácticas de atención que les es dedicado, por medio del análisis de cantidad y calidad. Los resultados han demostrado que los enfermeros realizan las acciones programáticas - prenatal, preventivo del cáncer uterino y planeamiento familiar, con mayor frecuencia que los médicos. Los médicos refieren que sus atendimientos se agotan en la consulta. Ambos profesionales afirman que orientan la prevención de la gravidez, DST/Sida y uso del preservativo. Casi la totalidad de los enfermeros y más del $50 \%$ de los médicos hacen acciones de intervención. Hay consenso en cuanto a la necesidad de implantación de un servicio sistematizado y que el municipio tendrá que capacitar a todos los profesionales, reestructurar los recursos materiales y humanos, insertar otros profesionales e integrar las acciones con servicios, además del de la salud.

Palabras clave: Adolescente. Programa Salude de la Familia. Prevención. Atención básica de salud. 